\title{
Multidimensional Time Model for Probability Cumulative Function and Connections Between Deterministic Computations and Probabilities
}

\author{
Michael Fundator \\ Board on BCSS of National Academy of Sciences, USA
}

Received: January 02, 2017 / Accepted: February 06, 2017 / Published: April 25, 2017.

\begin{abstract}
Multidimensional Time Model for Probability Cumulative Function can be reduced to finite-dimensional time model, which can be characterized by Boolean algebra for operations over events and their probabilities and index set for reduction of infinite dimensional time model to finite number of dimensions of time model considering also the fractal-dimensional time arising from alike supersymmetrical properties of probability. This can lead to various applications for parameter evaluation and risk reduction in such big complex data structures as complex dependence structures, images, networks, and graphs, missing and sparse data, such as to computer vision, biology, medicine, and various DNA analyses.
\end{abstract}

Keywords: finite-dimensional time model, normally distributed, complete class, Edgeworth's form, Chebyshev-Hermite polynomials.

"Let $\mathrm{Tp}$ denote a nonparametric multivariate test in $p$ dimensions. Let $\pi_{p \rightarrow q}$ denote a method of reducing the dimensionality to $\mathrm{q}$, such as by using the first q principal components, or by multidimensional scaling, or by "lining" or "planning". The q-dimensional form of the multivariate test might stil be applicable to the resulting q-dimensional data... test $\operatorname{Tq}\left(\pi_{p \rightarrow q}\right)$...conjecture that, when $\mathrm{p}$-dimensional data is sparse, $\mathrm{Tq}\left(\pi_{p \rightarrow q}\right)$ will often be in some sense a better test than Tp... this simple suggestion should not be confused with the method of "projecting" down to one dimension (lining), and then using a univariate test... it is best to think of $q$ as at least 2. The suggestion is so simple that it has probably been suggested several times, but I'd like to know of a reference. ("The Good Book" v.2 C356. Multivariate Tests after dimensionality reduction p. 277)

Corresponding author: Michael Fundator, Board on BCSS of National Academy of Sciences, USA.

\section{Introduction}

It seems that the brief introduction of the historical sketch of the development of the Theory of Brownian Motion and some historical problems that very often overlooked of would be very helpful to make understanding of this work more clear:

(1) [32-36] On May 30th, some 218 years ago problem to decide if a number is a special value of a well known function was solved by Gauss, when he observed, purely computationally, that the constant that equal to reciprocal of Arithmetic-Geometric Mean of 1 and $\sqrt{2}$ and would be named Gauss constant, is equal to the value of elliptic integral $\mathrm{M}(1, \sqrt{2})$ $=1 / \mathrm{G}=1.19814023473 \ldots$

$\mathrm{G}=1 / \operatorname{agM}(1, \sqrt{2})=\frac{2}{\pi} \int_{0}^{1} \frac{d t}{\sqrt{1-t^{4}}}=0.83462684167 \ldots$

to eleven decimal places. He commented in his diary that this result"will surely open up a whole new field of analysis". G is related also to Gamma function of $1 / 4$ as $\mathrm{G}=\Gamma^{2}(1 / 4) / 2 \sqrt{2} \pi^{3}$, where $\Gamma^{2}(1 / 4) / 4 \sqrt{\pi}=$ 
$1.85407467=\mathrm{F}(\mathrm{e}, \theta)=\int_{0}^{\theta} \frac{d \theta}{\sqrt{1-e^{2} \sin ^{2} \theta}}$ for $\mathrm{e}=\sin 45^{\prime}=$ $1 / \sqrt{2}$ and $\theta=90^{\prime}=\pi / 2$ is another constant and can be used in calculation of the lemniscate constants, $\mathrm{L}_{1}=$ $\pi \mathrm{G}$ and $\mathrm{L}_{2}=1 / 2 \mathrm{G}$ that can further be used in

(2) $[32,34,38-40]$ The theory of harmonic oscillators and dynamical systems through complex multiplication of elliptic curves that were used in 1859 by Hermite to discover number following Gauss' conjecture

$e^{\pi \sqrt{ } 163}=12^{3}\left(231^{2}-1\right)^{3}+743.99999999999925007 \ldots$ and

(3) Probabilistic approach to deterministic equations such as a question of assigning probability measure $p(x)$ over the solution of a linear system of equations $\mathrm{Ax}=\mathrm{b}$ if $\mathrm{A}$ and $\mathrm{b}$ are known, use of probability in statistics to remove "noise" from "signal". Would the measure be related to the concept of randomness (aleatory uncertainty), or would it be quantifying epistemic uncertainty, attributed to missing information? [29, Aleatory and Epistemic Uncertainty Civil Engineering - University of ... www.ce.memphis.edu/../C11.\%20\%20Aleatory\%20and $\% 20$ Epistemic\%20Uncertaint..]

(4) Connections between deterministic computations and probabilities have a long history. [37] The theorem of Erdös and Kac that the number of distinct prime factors in a randomly chosen large integer $n$ is approximately normally distributed with mean and variance $\log \log \mathrm{n}$, can be viewed without concept of epistemic uncertainty.

(5) [5] However, besides their approach we can also emphasize an example by A.Y. Khinchin, that the direct consequence of the Theorem of Lagrange, which says, that every natural number can be expressed as the sum of at most four squares, is that, if four sequences

$0,1^{2}, 2^{2}, 3^{2}, \ldots, \mathrm{k}^{2}, \ldots .$.

are added together, the resulting sequence contain all natural numbers, it was his main introductory point for Waring's Conjecture. Probabilistic and deterministic methods for inference on physical quantities have shared dualities from very early on: Legendre introduced the method of least squares in 1805 as a deterministic best fit for data without a probabilistic interpretation. Gauss' 1809 probabilistic formulation of the exact same method added a generative stochastic model for how the data might be assumed to have arisen. Legendre's least-squares is a useful method without the generative interpretation, but the Gaussian formulation adds the important notion of uncertainty (also interpretable as model capacity) that would later become crucial in areas like the study of dynamical systems. "It has been customary certainly to regard as an axiom the hypothesis that if any quantity has been determined by several direct observations, made under the same circumstances and with equal care, the arithmetical mean of the observed values affords the most probable value, if not rigorously, yet very nearly at least, so that it is always most safe to adhere to it." - Gauss (1809, section 177) Theoria motvs corporvm coelestivm in sectionibvs conicis Solem ambientivm [Theory of the Motion of the Heavenly Bodies Moving about the Sun in Conic Sections].

(6) Another open problem is a question of normality, if the decimal expansions of $\pi$, e, $\sqrt{ } 2, \sqrt{ } 10$, and many other mathematical constants such as $\gamma$, or $\log 2$ all have the property that the limiting frequency of any digit is one tenth, or any $n$-long string of decimal digits is $10^{-\mathrm{n}}$ (and similarly for binary expansions).

There are following examples for decimal expansion of $\pi$ from Weisstein, E. W. "Pi Digits. From MathWorld--A Wolfram Web Resource. http://mathworld.wolfram.com/PiDigits.html:

The sequence 0123456789 occurs beginning at digits 17387594880, 26852899245, 30243957439, 34549153953 , 41952536161, and 43289964000.

The sequence 9876543210 occurs beginning at digits 21981157633, 29832636867, 39232573648, 42140457481 , and 43065796214 .

The sequence 27182818284 (the first few digits of e) 
occurs beginning at digit 45111908393.

J. Havil in his book "The Irrationals" gives this impressive example, that sequence 0123456789 appears for the first time starting at the $17,387,594,880$ th digit; whereas 0691143420 continues to prove elusive. His book has ISBN 978-069114342-2 and was published 2 years ago by Princeton University Press. The website gives the record for this year as 10 trillion, with current of 13.3 trillion.

This problem is very much related to such open problems in the Theory of Brownian Motion as if all Brownian paths are possible, and the like problems of transience and recurrence of Random walk in 2 or more dimensions $[$ Peter Morters and Yuval Peres, Brownian Motion, CUP] [8-12, 14, 17].

This is closely related to the following questions and phenomena in the theory of multidimensional Brownian motion.

\section{Various Properties of Multidimensional Brownian Motion [8-12,14,17]}

First are listed various results, which are following from the general theory of Brownian motion.

(1) Theorem 1 (Levy 1940). Almost surely, $\mathrm{L}_{2}$ (B[0; 1]) $=0$.

The range of planar Brownian motion has zero area

Suppose $\{\mathrm{B}(\mathrm{t}): \mathrm{t} \geq 0\}$ is planar Brownian motion. Denote the Lebesgue measure on $\mathrm{R}^{\mathrm{d}}$ by $\mathrm{L}_{\mathrm{d}}$.

The Theorem 1 states that almost surely, $\mathrm{L}_{2}(\mathrm{~B}[0 ; 1])$ $=0$.

(2) Theorem 2 For $\mathrm{d}=2$, almost surely, the intersection of any finite number $p$ of in-dependent Brownian paths in $\mathrm{Rd}$ is nontrivial, i.e. contains points other than a possible common starting point.

(3) Theorem 3 A fixed two-dimensional projection of a three-dimensional Brownian motion is almost surely neighborhood recurrent.

(4) Theorem 4 Three-dimensional Brownian motion hits any infinite cylinder with probability 1 , but it does not hit all cylinders. There almost surely exists avoided infinite cylinder.

(5) The problem of finding lower bounds for the Hausdorff dimension of the intersection sets is best approached using the technique of stochastic co-dimension, which is to take a suitable random test set, and check whether it is zero or positive. This approach is based on using the family of percolation limit sets as test sets.

(6) [3] Brownian motion according to R. von Mises in "Probability, Statistics and Truth" page 186: "About a hundred years ago, the English botanist Brown observed under the microscope that certain organic liquids contain small particles moving to and fro in an incessantly agitated manner. It was discovered later that this so-called 'Brownian motion' is common to all sufficiently small particles suspended in a gas or in a liquid, and that it represents a mass phenomenon following the laws of probability calculus. Since we are only interested in the fundamental logical structure of this problem, we can simplify our conception by considering a two-dimensional scheme. We assume that the particles move in a zigzag course in the horizontal plane, excluding any up or downward motion, or else, we may say that we consider only the projection of the three-dimensional motion onto a horizontal plane."

It is clearly seen from the above examples, that the decimal expansion of $\pi$ does not follow strictly Gaussian distribution, but instead is supposed to follow some kind of slightly non-Gaussian distribution.

\section{Insights Into Near-Gaussian Distributions and d-dimensional Projections}

[4] Before considering any discussion about the above phenomenon or any possible approach to analyze or investigate it, it seems appropriate to quote Karl Pearson, who wrote 110 years ago on p. 189 "My custom of terming the curve the Gauss-Laplacian or normal curve saves us from proportioning the merit of discovery between the two great astronomer mathematicians." One of the definitions of Peirce of 
"normal" as of what would, in the long run, occur under certain circumstances, clearly implies Principle of prediction and LLN $[2,7,11]$.

"It is undeniable that, in a large number of important applications, we meet distributions which are at least approximately normal. Such is the case, e.g., with the distributions of errors of physical and astronomical measurements, a great number of demographical and biological distributions, etc." $[\mathrm{H}$. Cramer. Mathematical Methods of Statistics p.231].

[30] The first investigation of slightly non-Gaussian distributions was undertaken by Chebyshev around a century and a half ago, who studied in detail a family of orthogonal polynomials which form a natural basis for the expansions of these distributions. A few years later the same polynomials were also investigated by Hermite and they are called Chebyshev-Hermite or simply Hermite polynomials, their definition was first given by Laplace.

These methods use Edgeworth's form that is equivalent to the Gram-Charlier Type A series with use cumulant analysis for the representation of the distribution function in terms of different types of sums of functions of Gaussian processes.

A standard method of exploring high-dimensional datasets is to examine various low-dimensional projections thereof. In fact, many statistical procedures are based explicitly or implicitly on a projection pursuit. Under weak regularity conditions on a distribution $P=P(n)$ on $\mathrm{R}^{\mathrm{n}}$, most $d$-dimensional orthonormal projections of $P$ are similar (in the weak topology) to a mixture of centered, spherically symmetric Gaussian distributions on $\mathrm{R}^{\mathrm{d}}$ if $\mathrm{n}$ tends to infinity while $d$ is fixed.

\section{Analysis of Axioms of Probability of Von Mises [6,7, 11]}

Theory of von Mises was based on 2 axioms:

(1) $\forall \varepsilon_{i}$ (element appering in the sequence) $\varepsilon_{1}, \varepsilon_{2} \ldots \varepsilon_{i} \ldots ., \varepsilon_{i}$ has limiting frequency depending on $\varepsilon_{i}$
(2) For $\forall\left(\tau_{1}, \tau_{2}, \ldots \tau_{i} ..\right)$ ( possibly infinite subseq of). $\in\left(\varepsilon_{1}, \varepsilon_{2} \ldots \varepsilon_{i} \ldots.\right)$, with other selection method than prior knowledge of the values of elements selected, the limiting frequencies should be the same.

Property 1. is known as the LLN which in measure-theoretic probability theory is a theorem, holding for almost all sequences $x$.

Property 2. stands for the rules for selection method that called "selection rules", and selection rules that are different from "prior knowledge" are called "proper selection rules" in contrast to "improper".

After 18 years of debates, the logician Alonzo de Church proposed additional relation to the von Mises system of axioms that only "effectively calculable " selections should be admitted and thus the set of admissible place selections should consist of the "computable" or "partial recursive functions". With this addition of central notion of "recurrence" to the system of axioms of von Mises was introduced the Theory of Algorithms (or Recursive Function Theory or Computability Theory). "Kolmogorov-Loveland stochasticity" selection rule that was introduced some 25 years later was very valuable addition.

[8, 10-12] Richard von Mises mentions "the four fundamental operations" on pages 38-58 of his book "Probability, Statistics and Truth":

1. Selection;

2. Mixing;

3. Partition; and

4. Combination,

the last 3 defined so as to correspond to usual addition, division, and multiplication rules, and the 1 st one is defined as the attributes unchanged and the sequence of elements reduced by place selection, which correspond to the unchanging of distribution, and can be viewed as identity element and related to the previous operations.

Proposition1. Analysis, of how the integration over the events would work in the system of axioms of probability of von Mises, is leading to the decision, that it would definitely be an algebra with operations 
summation and multiplication. So we can assign ordinal number 4 to the general model.

$[5,8,10-12]$ Proposition3. Theorem5 Let $\mathrm{k}$ and 1 be arbitrary natural numbers. Then there exists a natural number $n(k, l)$ such that, if an arbitrary segment, of length $n(k, 1)$, of the sequence of natural numbers is divided in any manner into k classes (some of which may be empty), then an arithmetic progression of length 1 appears in at least one of these classes (Van der Warden's Theorem) (Khinchin "Three pearls of Number theory").

\section{Introduction of Multidimensional Time Model for Probability Cumulative Function}

(1) It follows from Proposition 3 (Van der Warden's Theorem) that infinite number of dimensions can be divided into some number that would depend on the properties of investigated model.

It is interesting to consider such point that Chemical analysis of escaping by particle energy barrier from potential hole is related to study of chemical equilibria. According to Le Chatelier's Principle for a state of dynamic equilibrium, a change in concentration, pressure or temperature of reactants in an equilibrated system shifts chemical equilibria toward the products or reactants, depending on reaction type, whether it is endothermic or exothermic. After it was used by Kramers this is closely related to the following questions and phenomena in the theory of multidimensional Brownian motion that multidimensional pattern could be related to time dimensions through the introduction in the theory of Brownian motion by $\mathrm{E}$ i $\mathrm{n} \mathrm{s}$ t e i $\mathrm{n}$ the consideration of a range of time intervals $\tau$, the possibility of a term proportional to $\tau$ in the expression for Moments of Brownian motion $B_{\tau}^{n}(\mathrm{n}>1)$ is related to the fact that the values of $X$ at moments $t_{1}, t_{2} \ldots t_{n}$ which lie sufficiently close together are no longer independent; and Moments of Brownian motion $B_{\tau}^{n}(\mathrm{n}>1)$ in fact are represented by a volume integral

$$
\int \ldots \int X\left(\mathrm{t}_{1}\right) X\left(\mathrm{t}_{2}\right) \ldots X\left(\mathrm{t}_{\mathrm{n}}\right) d \mathrm{t}_{1} d \mathrm{t}_{2} \ldots d \mathrm{t}_{\mathrm{n}}
$$

over an n-dimensional cube; the contribution to this integral due to a narrow cylinder extending along the diagonal $\mathrm{t}_{1}=\mathrm{t}_{2}=\ldots=\mathrm{t}_{\mathrm{n}}$ may give a term proportional to $\tau[15,16]$.

(2) Second approach to multidimensional time model through Cumulant Functions and time series analysis.

To strengthen this notion consider cumulants properties for time series analysis that provide measure of Gaussianity. If r.v. $\mathrm{X}$ is normal, then $\operatorname{cum}_{\mathrm{k}}\{\mathrm{X}\}=0$ for $\mathrm{k}>2$, where $\operatorname{cum}_{\mathrm{k}}$ denotes the joint cumulants of $\mathrm{X}$ with itself $\mathrm{k}$ times.

For simplicity consider seq of iid $\mathrm{X}_{\mathrm{i}}$ with all moments and $\mathrm{E}\left\{\mathrm{X}_{\mathrm{i}}\right\}=0$ and $\operatorname{var}\left\{\mathrm{X}_{\mathrm{i}}\right\}=1$, then for $\mathrm{S}_{\mathrm{n}}=$ $\sum \mathrm{X}_{\mathrm{i}} / \sqrt{n}$ cum $_{\mathrm{k}}\left\{\mathrm{S}_{\mathrm{n}}\right\}=$ ncum $_{\mathrm{k}}\{\mathrm{X}\} / \mathrm{n}^{\mathrm{k} / 2}$ that tends to 0 for $\mathrm{k}>2$, as $\mathrm{n}$ tends to infinity, so $\mathrm{S}_{\mathrm{n}}$ has a limiting normal distribution.

And for time series analysis the moment function

$\mathrm{E}\left\{\mathrm{X}\left(\mathrm{t}+\mathrm{u}_{1}\right) \ldots \mathrm{X}\left(\mathrm{t}+\mathrm{u}_{\mathrm{k}-1}\right) \mathrm{X}(\mathrm{t})\right\}$ would not depend on $\mathrm{t}$, and on the short time interval centered at point of time $\mathrm{t}$ can be approximated by normal distribution [12].

(3) Additional to the Brownian motion considerations in the theory of chemical reactions and time series analysis for cumulant functions, the same results can be obtained from the consideration of associated random variables.

Definition1 For $n>1$ the set of $\mathrm{rv} \mathrm{X}_{\mathrm{i}}$ is said to be associated, if for all given real-valued functions $\mathrm{g}_{\mathrm{i}}$ that are increasing in each component when the other components are held fixed, the inequality

E $\quad\left[\prod_{j}^{2} g_{j}(\mathrm{X})\right] \geq \prod_{j}^{2} E \quad\left(\mathrm{~g}_{\mathrm{j}}(\mathrm{X})\right)$ holds, or equivalently, Corr $\left(\mathrm{g}_{\mathrm{i}}(\mathrm{X}), \mathrm{g}_{\mathrm{j}}(\mathrm{X})\right) \geq 0$,

Esary, et al. proved the following theorem:

Theorem6 (a) A set consisting of a single random variable is a set of associated random variables.

(b) Independent random variables are associated random variables. (c) A subset of a set of associated random variables forms a set of associated random variables. (d) Increasing functions of associated random variables are associated random variables [12]. 
Proposition 4. Therefore, the process $X(t)$ with above properties can be represented by composition of Brownian motion processes in finite-dimensional time model $[8,10,12,13]$.

Next consider Stone representation of Boolean algebra, which is represented by an algebra with known axioms for Boolean algebra and can be characterized by quadruplets $\mathrm{B}=<\mathrm{X}, 0, *, \sim>$, where 0 is an element from a set $\mathrm{X}$, and $*$ is a binary operation and $\sim$ is an unary operation, which would be a Boolean algebra with 1 as a unit on the operations $\Lambda, \vee$, and $\sim$. Besides that it has four unary operations, two of which are constant operations, another is the identity, and negation and besides the number of n-ary operations, the number of the dimensions that infinite-dimensional model can be reduced to through application of Boolean prime ideal theorem and Stone duality, can be indexed by an index set.

Proposition 5 Theorem7 (belongs to the author). Multidimensional Time Model for Probability Cumulative Function can be reduced to finite-dimensional time model, which can be characterized by Boolean algebra for operations over events and their probabilities and index set for reduction of infinite dimensional time model to finite number of dimensions of time model considering also the fractal-dimensional time arising from alike supersymmetrical properties of probability.

\section{Application of Sampling and Functional Inequalities to Multidimensional Considerations}

(1) [18-24] Hilbert some 120 years ago, though did not use Cauchy's inequality, but used rather some complicated methods to prove that $\sum \sum \frac{a_{m} b_{n}}{m+n} \leq$ $C\left(\sum a_{m}^{2}\right)^{1 / 2}\left(\sum b_{m}^{2}\right)^{1 / 2}$, with $\mathrm{C}=2 \pi$, where the summations run from 1 to $+\infty$, which was later reduced by $\mathrm{I}$. Schur to $\mathrm{C}=\pi$. There were some improvements to constant $\mathrm{C}$ in Hilbert's inequality such, as by H. Frazer, who showed that it can be reduced to $(\mathrm{n}+1) \sin \frac{\pi}{(\mathrm{n}+1)}$,

and N. G. de Bruijn and H. S. Wilf showed that the best possible constant for the discrete case of summations running from 1 to $\mathrm{N}$ in $\sum \sum \frac{a_{m} a_{n}}{m+n} \leq C\left(\sum a_{n}^{2}\right)^{1 / 2}$ can be replaced by

$$
\mathrm{C}_{\mathrm{N}}=\pi-\frac{1}{2} \pi^{5}(\log \mathrm{N})^{-2}+\mathrm{O}\left(\log \log (\log \mathrm{N})^{-3}\right), \mathrm{N} \rightarrow+\infty .
$$

These results are clearly related to Sampling Theory, and the form of the constants are pointing out that such sampling can be considered as it is made from a multidimensional sample.

$[28,8,10,12]$ The later remarkable result is very important in light of the Bruijn-Wilf type best constant for $\mathrm{n}^{\text {th }}$ finite section of Carleman's inequality

$$
\begin{aligned}
& \sum_{k=1}^{n}\left(a_{1} a_{2} \ldots a_{k}\right)^{1 / k}<\mathrm{C}_{\mathrm{n}} \sum_{k=1}^{n} a_{k} . \\
& \mathrm{C}_{\mathrm{n}}=\mathrm{e}-2 \pi^{2} \mathrm{e} 1 / \ln (\mathrm{n})^{2}+\mathrm{O}\left(1 / \ln (\mathrm{n})^{3}\right) \\
& \mathrm{C}_{2}=1 / 2(1+\operatorname{sqr}(2)), \mathrm{C}_{3}=4 / 3 . \text { And for the Hardy's }
\end{aligned}
$$
inequality

$$
\mathrm{C}_{\mathrm{n}}=4-16 \pi^{2} / \ln (\mathrm{n})^{2}+\mathrm{O}\left(\ln \ln \mathrm{n} / \ln (\mathrm{n})^{3}\right),
$$

The extensions of Widder with the remark of Hardy are the following inequalities:

$$
\begin{aligned}
& \sum \sum \frac{\log (\boldsymbol{m} / \boldsymbol{n})}{\boldsymbol{m}-\boldsymbol{n}} \boldsymbol{a}_{\boldsymbol{m}} \boldsymbol{b}_{\boldsymbol{n}} \leq \boldsymbol{\pi}\left(\sum \sum \frac{\boldsymbol{a}_{\boldsymbol{m}} \boldsymbol{a}_{\boldsymbol{n}}}{\boldsymbol{m} \boldsymbol{n} \boldsymbol{n}}\right)^{\mathbf{1} / \mathbf{2}}\left(\sum \sum \frac{\boldsymbol{b}_{\boldsymbol{m}} \boldsymbol{b}_{\boldsymbol{n}}}{\boldsymbol{m}+\boldsymbol{n}}\right)^{\mathbf{1} / \mathbf{2}} \\
& \sum \sum \frac{|\log (m / n)|}{\max (m, n)} a_{m} b_{n}
\end{aligned}
$$

$2\left(\sum \sum \frac{a_{m} a_{n}}{\max (m, n)}\right)^{1 / 2}\left(\sum \sum \frac{b_{m} b_{n}}{\max (m, n)}\right)^{1 / 2} \quad$ where the summations run from 0 to $+\infty$, and the coefficient on the left-hand side is interpreted as $1 / \mathrm{n}$ when $m=\mathrm{n}$.

The striking difference in the constants $\pi$ and 2 would be summarized later in Proposition6

(2) $[8,10,12,27]$ In this form it is very much resembling the famuos Landau-Kolmogorov inequality, which was inroduced by E. Landau 2 years after I. Schur's improvement for Hilbert's inequality that for $\|f\|$ to be the supremum of $|f(x)|$, a real-valued function $f$ defined on $(0, \infty)$. and for a usually 
defined norm of real-valued function $f$ defined on $(0, \infty) \quad$ as $\quad\|f\|=\sqrt{\int_{0}^{\infty}[f(x)]^{2} d x}$. there are different constants $C(n, k)$ in inequality $\left\|f^{(k)}\right\| \leq C(n, k)\|f\|^{1-k / n}\left\|f^{(n)}\right\|^{k / n}$.

[8, 10, 12, E. F. Beckenbach and R. Bellman, "Inequalities," Springer] Similar notions about multidimensional nature of constants can arise in the consideration of discrete inequalities of Fan, Taussky, and Todd that were introduced some 50 to 60 years ago.

(3) $\sum_{i=0}^{n-1}\left(x_{i} \quad-2 \quad x_{i+1}+x_{i+2} \quad\right)^{2} \geq 16$ $\sin ^{4} \frac{\pi}{2(n+1)} \sum_{i=0}^{n} x_{i}^{2}$;for the sequence of real numbers $\mathrm{x}_{0}=0, \mathrm{x}_{1}, \ldots . \mathrm{x}_{\mathrm{n}}, \mathrm{x}_{0}=\mathrm{x}_{\mathrm{n}+1}=0$, with equality holding if and only if $\mathrm{x}_{\mathrm{i}}=\operatorname{csin} \frac{i \pi}{n+1}, \mathrm{i}=1, \ldots, \mathrm{n}$, where $\mathrm{c}$ is a real constant and for the sequence of real numbers $\mathrm{x}_{1}=0$, $\mathrm{x}_{1}, \ldots . . \mathrm{x}_{\mathrm{n}}: \sum_{i=1}^{n-1}\left(x_{i}-x_{i+1}\right)^{2} \geq 4 \sin ^{2} \frac{(i-1) \pi}{2 n-1} \sum_{i=2}^{n} x_{i}^{2}$.

(4) $[8,10,12,25,26] 99$ years passed before V. Milman established reverse form of the Brunn-Minkovski inequality that was proposed by Hermann Brunn 1 year before Hilbert's inequality.

$[8,10,12]$ PROPOSITION6. Therefore, the use of supremum of a function over an interval, or maximum, or minimum instead of sum of the numbers completely loses the notion of multidimensionality and reduces it possibly to 1 dimension instead of double-number dimensional.

\section{Support from and application to scientific theories requiring complex data analysis.}

Application of similarities in the different theories of chemical reactions rates to DNA analysis.

Rice-Ramsperger-Kassel-Marcus (RRKM) rate theory developed about the time of Bohr, Kramers, and Slater (BKS) theory that uses notions of active and inactive molecules is a good introduction to the use of well developed mathematical apparatus of Boolean algebra and fuzzy logic. Kramers reaction rate theory predicts that the solvent dynamics will always decrease the reaction rate, or that in the most favorable situation no barrier recrossings take place and the rate constant corresponds with that of Rice-RamspergerKassel-Marcus (RRKM) rate theory, which gives the Transition State Theory rate as a function of the collision rate for independent polyatomic molecules.

Based on both theories BKS and RRKM that depend on time scale time scale of the correlations in the random solvent fluctuations and of barrier escaping of a particle.

[12] The following is the author's idea.

Since RRK theory is based on the following assumptions:

(1) A molecule is considered as a collection of $s$ coupled harmonic oscillators.

(2)The intermolecular distribution of the excess energy (IVR) occurs faster thanthe unimolecular decomposition of the activated complex back to reactants (referred to as the "ergodic assumption");

the same approach can be used for DNA and RNA analysis.

In Deoxyribonucleic acid (DNA) analysis most DNA molecules consist of two biopolymer strands coiled around each other to form a double helix. The two DNA strands are termed polynucleotides since they are composed of simpler monomer units called nucleotides Each nucleotide is composed of one of four nitrogen-containing nucleobases - either cytosine $(\mathrm{C})$, guanine $(\mathrm{G})$, adenine $(\mathrm{A})$, or thymine $(\mathrm{T})$ - and a sugar called deoxyribose and a phosphate group. The nucleotides are joined to one another in a chain by covalent bonds between the sugar of one nucleotide and the phosphate of the next, resulting in an alternating sugar-phosphate backbone.

Application to computer vision, biology, medicine $[8,10,12]$.

In computer vision, the target probability $\mathrm{p}(\mathrm{x})$ is often defined on a graph representation $\mathrm{G}=<\mathrm{V}, \mathrm{E}>$, 
thus can be divided in two types of graph structures, and thus the Markov chains are designed accordingly.

1. Descriptive models on a plat graph where all vertices are semantically at the same level, e.g. various Markov random fields image segmentation, graph partition/coloring, shaping

2. Generative models on a hierarchic And-Or graph with multiple levels of vertices

where a high level vertex is divided into various components at the low level, e.g. Markov trees, sparse coding, object recognition, image parsing, etc

In advanced models, these two structures are integrated because the vertices at each level of a generative model are connected by contextual horizontal links which represent various relations among the vertices.

These computer vision simulations are considered very important for DNA structures representation, as could be clearly seen from the following examples. The biomedical applications of fractal concepts have led to a wealth of new insights in biology and physiology, including a new formulation of the concept of health. The complexity inherent in physiological structures and processes has been described by random fractals. Fractal scaling in various physiological contexts contributed to the analysis of the DNA sequencing, the dendritic branching of neurons and blood vessels, the mammalian lung, the beating of the heart, the dynamics of proteins, ion channel gating and radioactive clearance curves from the body in order to reveal an underlying unity to physiological processes.

\section{References}

[1] Gauss (1809, section 177) Theoria motvs corporvm coelestivm in sectionibvs conicis Solem ambientivm [Theory of the Motion of the Heavenly Bodies Moving about the Sun in Conic Sections]

[2] Peirce, Charles S. (c. 1909 MS), Collected Papers v. 6.

[3] Richard von Mises "Probability, Statistics, and Truth" Dover/81 ISBN: 978-0-486-24214-9

[4] Karl Pearson, Das Fehlergesetz und seine Verallgemeinerungen durch Fechner und Pearson. A rejoinder". Biometrika 4 (1): 169-212

[5] Khinchin, Three pearls of Number theory.

[6] Alonso de Church "On the concept of a random sequence"

[7] P. Erdos "On the strong Law of Large Numbers"

[8] M. Fundator Applications of Multidimensional Time Model for Probability Cumulative Function for Parameter and Risk Reduction in JSM Proceedings Health Policy Statistics Section.

[9] W. Feller "Introduction to Probability Theory" v-1, 2, Wiley/99 ISBN: 978-81-265-1805-

[10] M. Fundator Multidimensional Time Model for Probability Cumulative Function in JSM Proceedings Health Policy Statistics Section

[11] M. Fundator Testing Ststistical Hypothesis in Light of Mathematical Aspects in Analysis of Probability doi:10.20944/preprints201607.0069.v1

[12] M. Fundator Application of Multidimensional time model for probability Cumulative Function to Brownian motion on fractals in chemical reactions. In publication.

[13] L. Dumbgen, P. D. Conte-Zerial On low-dimensional projections of high-dimensional distributions.

[14] Adelman, Burdzy and Pemantle (1998): Sets avoided by Brownian motion.Ann. Probab. 26, 429-464.

[15] M. P. Bianchi A BGK-type model for a gas mixture undergoing reversible reaction.

[16] H. A. Kramers, Brownian motion in a field of force and the diffusion model of chemical reactions. Physica, 7,4, 284-304 (1940)

[17] P. Morters Lecture notes.

[18] Hilbert D., Uber die Darstellung definiter Formen als Summe von Formenquadraten, Math Ann. 32, 342-350 (88).

[19] I. Schur, Bemerkungen zur Theorie der beschränkten Bilinearformen mit unendlich vielen Veränderlichen, J. reineangew. Math., 140 (11)

[20] D. V. Widder, An inequality related to one of Hilbert's, J. London Math. Soc. 4 ,194-198.

[21] Frazer C. Note on Hilber's inequality, J. London Math. Soc. $21 / 46$ pp..7-9

[22] G. H. Hardy, J. E. Littlewood and G. P'olya, Inequalities, 2nd ed., CUP, Cambridge, 1988

[23] Mitrinovic, Dragoslav S. Analytic Inequalities.

[24] H.S. Wilf, Finite sections of some classical inequalities, Ergebnisse der Mathematik, Band 52, Springer

[25] Minkowski, Hermann (1896). Geometrie der Zahlen. Leipzig: Teubner.

[26] Milman, Vitali D. (1986). "Inégalité de Brunn-Minkowski inverse et applications à la théorie locale des espacesnormés. [An inverse form of the Brunn-Minkowski inequality, with applications to the localtheory of normed spaces]". C. R. Acad. Sci. Paris 


\section{Between Deterministic Computations and Probabilities}

Sér. I Math. 302 (1): 25-28

[27] Landau, E. (1913). "Ungleichungen für zweimal differenzierbare Funktionen". Proc. London Math. Soc. 13:43-49. doi:10.1112/plms/s2-13.1.43

[28] N. G. de Bruijn Carleman's Inequality for finite series. Koninkl. Nederl. Akademie. Proceedings Series A,66,4

[29] Philipp Hennig, Michael A Osborne, and Mark Girolami Probabilistic Numerics and Uncertainty in Computations rspa.royalsocietypublishing.org

[30] S.Blinnikov, R.Moessner Expansions for nearly Gaussian distributions Astrophysics

[31] Halmos, Paul Lectures on Boolean Algebras. van Nostrand.

[32] Gauss, C. F. Werke. Gottingen-Leipzig, 1868-1927.

[33] David A. Cox, The arithmetic-geometric mean of Gauss. L'Enseignement Math'ematique, 30(1984), 275-330.

[34] Vladimir G. Tkachev Elliptic functions: Introduction course http://www.math.kth.se/ 'tkatchev
[35] Levi W. Meech Short Method of Elliptic Functions The Analyst, Vol. 4, No. 5 (Sep., 1877), pp. 129-136. Annals of Mathematics http://www.jstor.org/stable/2635660

[36] Milton Abramowitz, Irene A. Stegun Handbook of Mathematical Functions: with Formulas, Graphs, and Mathematical.

[37] Erdős, Paul; Kac, Mark "The Gaussian Law of Errors in the Theory of Additive Number Theoretic Functions". American Journal of Mathematics. 62 (1/4): 738-742.

[38] Dorian Goldfeld, Gauss's class number problem for imaginary quadratic fields, Bull. Amer. Math. Soc. (N.S.) 13 (1985), no. 1, 23-37. https://doi.org/10.1090/S0273-0979-1985-15352-2

[39] Barrow, John D The Constants of Nature. London: Jonathan Cape. ISBN 0-224-06135-6.

[40] Stark, H. M. (1969), "On the gap in the theorem of Heegner" Journal of Number Theory, 1: 16-27, doi:10.1016/0022-314X(69)90023-7 